\title{
Midcarpal Hemiarthroplasty for Wrist Arthritis: Rationale and Early Results
}

\author{
Scott W. Wolfe, M.D. ${ }^{1}$ \\ ${ }^{1}$ Department of Hand and Upper Extremity Surgery, Hospital for \\ Special Surgery, New York, New York \\ 2 Department of Orthopedic Surgery, Southend University Hospital, \\ Essex, United Kingdom \\ ${ }^{3}$ Department of Orthopaedics, Warren Alpert Medical School of \\ Brown University and Rhode Island Hospital, Providence, Rhode \\ Island
}

Michael C. Vance, M.D. ${ }^{1}$ Greg Packer, M.D. ${ }^{2}$ David Tan, M.D. ${ }^{1} \quad$ J.J. Trey Crisco, Ph.D. ${ }^{3}$

J Wrist Surg 2012;1:61-68.

\begin{abstract}
Address for correspondence and reprint requests Scott W. Wolfe, M.D., Hospital for Special Surgery, 535 East 70th Street, New York, NY 10021 (e-mail: WolfeS@hss.edu).
\end{abstract}

Midcarpal hemiarthroplasty is a novel motion-preserving treatment for radiocarpal arthritis and is an alternative to current procedures that provide pain relief at the expense of wrist biomechanics and natural motion. It is indicated primarily in active patients with a wellpreserved distal row and debilitating arthritic symptoms. By resurfacing the proximal carpal row, midcarpal arthroplasty relieves pain while preserving the midcarpal articulation and the anatomic center of wrist rotation. This technique has theoretical advantages when compared with current treatment options (i.e., arthrodesis and total wrist arthroplasty) since it provides coupled wrist motion, preserves radial length, is technically simple, and avoids the inherent risks of nonunion and distal component failure. The KinematX midcarpal hemiarthroplasty has an anatomic design and does not disrupt the integrity of the wrist ligaments. We have implanted this prosthesis in nine patients with promising early results. The indications for surgery were as follows: scapholunate advanced collapse wrist (three), posttraumatic osteoarthritis (three), inflammatory arthritis (two), and Keinböck disease (one). Prospective data has been collected and the results are preliminary given the infancy of the procedure. The mean follow-up was 30.9 weeks (range: 16 to 56 weeks). The mean Mayo wrist score increased from 31.9 preoperatively to $58.8(p<0.05)$ and the mean DASH score improved significantly from 47.8 preoperatively to 28.7 $(p<0.05)$. There was a trend toward increased motion but statistical significance was not reached. Two patients required manipulation for wrist stiffness. There was no evidence of prosthetic loosening or capitolunate narrowing. The procedure is simple (average surgical time was 49 minutes) and maintains coupled wrist motion through preservation of the midcarpal articulation. The preliminary data show that it appears safe but considerably longer follow-up is required before conclusions can be drawn as to its durability, reliability, and overall success. The level of evidence for this study is therapeutic level IV (case series).
Painful wrist arthritis is a debilitating condition for which current treatment options are limited. The primary goal of treatment for the symptomatic, arthritic wrist is to achieve a pain-free, functional, and stable joint that is able to bear load. This is ideally achieved while preserving wrist motion. The causes of wrist arthritis are varied and include the sequelae of trauma, carpal instability, Keinböck disease, and inflammatory arthropathy. ${ }^{1,2}$ The pattern and severity of articular involvement differ according to the etiology and guide the surgeon in recommending treatment. Ultimately, patients ISSN 2163-3916. 
that remain symptomatic after exhausting conservative options are indicated for one of the several operative procedures, loosely grouped into ablative surgery, arthrodesis, or arthroplasty.

Ablative procedures are those in which the pain-generating segment of the arthritic joint is excised. Radial styloidectomy, partial scaphoidectomy, and proximal row carpectomy (PRC) are the more common ablative procedures performed, with PRC being a recognized salvage operation for scapholunate advanced collapse (SLAC) and scaphoid nonunion advanced collapse (SNAC). ${ }^{3-9}$ Ablative procedures, while oftentimes successful in relieving pain, can be complicated by resultant instability or progressive articular degeneration leading to further symptoms. ${ }^{9-13}$

Total wrist arthrodesis is a reliable operation for pain relief and is favored as a surgical option in the severely arthritic wrist because of its relative ease of execution, durability, and predictable long-term results. ${ }^{14-17}$ Pain relief, however, comes at the expense of wrist motion with notable functional limitations in personal hygiene, applying a forceful grip, picking up small objects, and working in tight spaces. ${ }^{18-20}$

Partial arthrodesis involves fusing specific segments of the wrist to preserve some degree of wrist motion while addressing symptomatic arthritic articulations. Limitations include variable success in fusion rates and the technical difficulties of fusing small carpal bones. Scaphoid excision and four-corner fusion is the most commonly performed limited arthrodesis as it preserves a functional arc of motion with satisfactory grip strength. ${ }^{21,22}$ Yet, the nonunion rate averages 5\%, with reported rates upwards of $25 \%$, and remaining radiolunate motion is primarily limited to the flexion-extension plane. $^{22,23}$ Radioscapholunate fusion is another partial arthrodesis for radiocarpal arthritis and is an attractive surgical option as it preserves critical midcarpal motion and carpal height. ${ }^{24}$ Retaining the midcarpal articulation allows for continued coupled wrist motion known as the "dart-thrower's" arc of radial-extension to ulnar flexion. ${ }^{25}$ Modifications to this procedure, including distal scaphoid and triquetral excision, have further improved the range of motion and overall outcomes. ${ }^{26-28}$ However, problems such as nonunion rates of 3 to $26 \%$, and progressive symptomatic midcarpal arthritis, are well documented. ${ }^{29-31}$

Wrist arthroplasty is an attractive motion-sparing concept for the treatment of arthritis and has evolved in multiple iterations over the past 40 years. Swanson first reported arthroplasty for the wrist in 1973, using a flexible silicone implant. ${ }^{32}$ Complications such as implant failure and silicone synovitis fueled the design of metal-on-polyethylene modular prostheses. ${ }^{33}$ Despite advances in design, total wrist prostheses continue to be complicated by instability and distal component failure. ${ }^{34-42}$ Nonetheless, total wrist arthroplasty is a viable option in a carefully selected patient with inflammatory arthritis of the wrist and low functional demands.

While the mechanics of the wrist remain incompletely understood, growing evidence has illustrated the importance of the dart-thrower's motion. Studies have demonstrated that the pattern of carpal bone motion associated with wrist movement along the dart thrower's path is unique. ${ }^{43}$ The mechanical axis of the wrist is not aligned with the anatomic axes of flexion-extension and radioulnar deviation, but is rather aligned with the dart thrower's axis of radial-extension to ulnar-flexion. ${ }^{44}$ It is hypothesized that all highly functional motions of the upper extremity utilize the dart thrower's motion. Its presence in many occupational, recreational, and household activities demonstrates the functional importance of this wrist motion. ${ }^{45}$

The dart-thrower's plane of coupled flexion-extension and radioulnar motion occurs predominantly at the midcarpal joint, suggesting the importance of this articulation in prosthetic design. ${ }^{24,43,46}$ Past designs of total wrist arthroplasty have largely replicated the contour and kinematics of the radiocarpal joint, thus constraining motion to the anatomical directions and minimizing the important dart thrower's arc. Further, excising portions of the distal radius relocates the center of rotation (COR) proximal to that of the normal wrist. There is a substantial clinical evidence that prosthetic failure is associated with failure to replicate normal joint kinematics in the spine, knee, shoulder, and the wrist. ${ }^{47-51}$ A proximal wrist center of rotation would increase the moment on the bone-cement interface of the distal component and may contribute to the high incidence of distal component loosening.

The concerning failure rate of current radiocarpal arthroplasty designs, and the improved understanding of coupled wrist motion, prompted the authors (S.W.W. and J.J.C.) to design a wrist replacement that would emulate the kinematics of the midcarpal joint. The KinematX (Extremity Medical, Parsippany, NJ) midcarpal hemiarthroplasty (-Fig. 1) is a monobloc component that replaces the proximal carpal row, while retaining the anatomic COR, the normal capsuloligamentous envelope, and preservation of midcarpal coupled motion. Conceptually, replacement of the arthritic proximal carpal row with a hemiarthroplasty implant is appealing in that it simultaneously preserves midcarpal motion and carpal height without the attendant risks of nonunion as seen in partial arthrodeses or the threat of distal component failure as documented in total wrist arthroplasty. Furthermore, the technique is potentially easier to execute and leaves recourse for either total wrist arthroplasty or arthrodesis should revision become necessary. The purpose of this article is to review the early outcomes of the first nine cases of midcarpal hemiarthroplasty performed for a variety of etiologies.

\section{Indications}

Midcarpal hemiarthroplasty is a motion-preserving alternative for the treatment of painful radiocarpal arthrosis that remains symptomatic and functionally limiting despite nonsurgical management. Indications include SLAC, SNAC, posttraumatic osteoarthritis, Keinböck disease, or inflammatory arthritis (-Fig. 2). Localized radial styloid osteoarthritis can be treated by less invasive means. The ideal patient for midcarpal hemiarthroplasty is an active patient whose midcarpal joint is relatively preserved. Patients with ipsilateral shoulder or elbow disease are also excellent candidates as the 


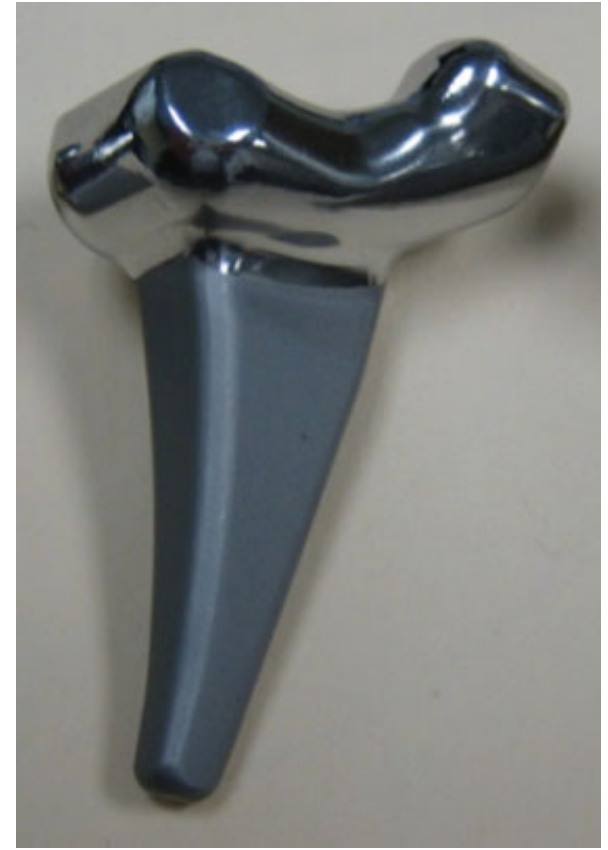

Figure 1 The KinematX prosthesis is an anatomical replacement of the proximal row which preserves the midcarpal articulation and wrist center of rotation.

preservation of wrist range of motion optimizes upper extremity function.

\section{Contraindications/Precautions}

Successful midcarpal hemiarthroplasty is determined more by individual patient selection than the underlying diagnosis. This technique is a ligament-sparing method that respects the complex wrist anatomy to provide coupled wrist motion. As such, the condition of the surrounding soft tissue envelope and underlying bony architecture is crucial. The capsuloligamentous sleeve of the wrist must be preserved and patients with severe soft tissue compromise may be at risk for resultant instability, excluding them as candidates for hemiarthroplasty. Patients with inflammatory disease and aggressive active synovitis are likewise at risk for instability or distal carpal row articular erosion, and may be better served by alternate procedures.

Midcarpal wrist hemiarthroplasty is a prosthetic replacement of the proximal row. This resurfacing procedure relies on the midcarpal articulation and to what extent midcarpal arthrosis precludes a good outcome remains unknown. It seems logical that advanced degenerative disease of the capitolunate joint is a contraindication to midcarpal hemiarthroplasty but early narrowing may be acceptable. Capitolunate narrowing in the setting of PRC or distal scaphoid excision has not been shown to compromise results; and the only published account of wrist hemiarthroplasty reports good pain relief despite degenerative changes of the capitate head. ${ }^{6,9,52}$

Durability of the hemiarthroplasty is dependent on prosthetic stability and thus the distal radius must be capable of

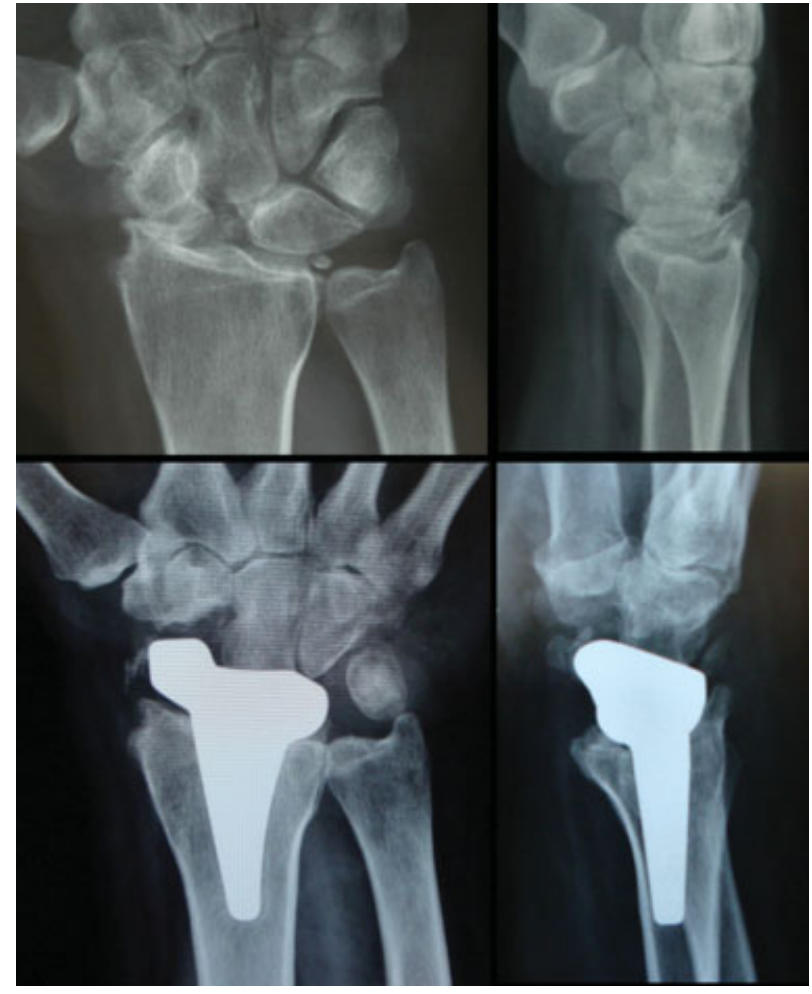

Figure 2 SLAC wrist in a 41-year-old active right hand dominant man. Preoperative images above; postoperative images below at 1 year after surgery. Resumed golfing on a regular basis at 12 weeks postoperatively. SLAC, scapholunate advanced collapse.

supporting the implant. Significant osteopenia, erosions due to inflammatory arthropathy, or exaggerated epiphyseal/ metaphyseal deformity may adversely affect implant fixation and lead to loosening.

Additional contraindications to hemiarthroplasty include recent or recalcitrant infection, previous surgical fusion, and lack of active wrist extension. Younger age and active lifestyle are not considered contraindications, as with total wrist arthroplasty, since activity restrictions are less stringent given that distal component loosening is not a concern.

\section{Surgical Technique}

The following technique is for implantation of the KinematX midcarpal hemiarthroplasty (Extremity Medical, Parsippany, $\mathrm{NJ}$ ) (-Table 1). Use of a radiographic template is recommended to preoperatively determine appropriate implant size. The templated implant should reproduce the size of the proximal carpal row and fit the distal radius on PA and lateral radiographs.

Routine skin preparation and upper extremity draping is conducted after the administration of preoperative IV prophylactic antibiotics. A padded tourniquet is applied to the upper arm. A dorsal longitudinal incision, 4 to $5 \mathrm{~cm}$ in length, is made over the wrist in line with the third metacarpal. Thick subcutaneous radial and ulnar skin flaps are elevated directly off the extensor retinaculum to minimize the risk of woundhealing problems. The extensor retinaculum is incised just 
Table 1 KinematX Midcarpal Hemiarthroplasty

\begin{tabular}{|c|}
\hline Pearls \\
\hline $\begin{array}{l}\text { Create a proximally based capsular flap } \\
\text { Leave a wafer of triquetrum attached to capsule to } \\
\text { anchor DRC and DIC } \\
\text { Preserve the origins of the palmar and dorsal extrinsic } \\
\text { wrist ligaments } \\
\text { Use the power rasp to prepare the surface of the distal } \\
\text { radius } \\
\text { Maintain rotational alignment during broaching }\end{array}$ \\
\hline Pitfalls \\
\hline $\begin{array}{l}\text { Failure to place the guidewire down the central axis of the } \\
\text { radius } \\
\text { Damage to the capitate head during instrumentation } \\
\text { "Overstuffing" leads to stiffness (small degree of laxity is } \\
\text { preferred) }\end{array}$ \\
\hline
\end{tabular}

DRC, dorsal radiocarpal ligament; DIC, dorsal intercarpal ligament.

radial to Lister's tubercle, reflected ulnarly, and the fourth dorsal compartment is elevated in continuity from the dorsum of the radius. At the discretion of the surgeon, the extensor pollicis longus (EPL) tendon can be freed from its sheath and transposed radially. Additional dissection of the radius is rarely required, as access to the intramedullary canal is facilitated by excision of the proximal carpal row. We prefer to preserve the posterior interosseous nerve as its role in wrist proprioception may prove valuable. ${ }^{53,54}$

A wide, proximally based rectangular dorsal capsular flap is created by incising the capsule beneath the tendons of the second and fifth dorsal compartments. The capsule is divided distally at the mid-capitate and reflected proximally, leaving a large capsular sleeve for later reattachment. Using an osteotome, a wafer of the triquetrum is raised in continuity with the capsule to preserve the attachments of the dorsal radiotriquetral and dorsal intercarpal ligaments.

With the joint exposed, the proximal row is excised. Removal of the scaphoid, triquetrum, and lunate can be accomplished in piecemeal fashion or en bloc using joysticks and sharp curved elevators. It is critical that the surgeon does not damage the palmar extrinsic ligaments or the cartilage surface of the capitate during removal.

The articular surface of the radius is exposed by wrist flexion and palmar translation of the distal row. Radial exposure is facilitated with insertion of a thin retractor under the volar lip. Particular care should be taken to protect the capitate articular surface at all times. The radius is first prepared using a custom elliptical power rasp that is contoured to the articular surface of the radius. The intent of the rasp is to remove the articular cartilage, flatten the interfacet ridge, and shape the subchondral plate while leaving the origins of the important extrinsic wrist ligaments fully intact dorsally and volarly. The tool is best used in a radial to ulnar and dorsal to volar sweeping motion. The subchondral bone provides support for the implant and should not be removed.

A $2.5 \mathrm{~mm}$ primary guidewire is inserted down the medullary canal, beginning $\sim 5 \mathrm{~mm}$ volar to the dorsal lip of the distal radius, or between the middle and dorsal thirds of the radial articular surface height. The ulnar border of Lister's tubercle is an anatomic landmark to guide proper placement in the coronal plane. Central positioning of the guidewire is verified under fluoroscopy.

Canal preparation begins with creating a window through the subchondral plate for broaching. A box chisel corresponding to the templated implant size is placed over the guidewire and impacted with the mallet until seated. The portion of the subchondral bone designated by the box chisel outline may be removed using small osteotomes or a high speed burr. The starter broach is impacted over the guidewire until flush with the subchondral bone. The canal is sequentially broached until the templated size is reached. Maintaining appropriate alignment during broaching is critical. Malrotation may cause the ulnar and radial borders of the implant to lift off of the supporting subchondral bone. The guidewire is removed once broaching is complete.

The trial component is impacted into position and the carpus is gently reduced onto the bearing surface of the implant, taking care to avoid injury to the capitate articular surface. Implant size, joint range of motion, and stability are assessed. In general, a degree of laxity is preferred over tension in the dorsal and volar ligaments, and will lead to more rapid return of motion. The final implant is gently impacted until it is fully seated on the contoured subchondral plate. Pressurized cementing is an option if compromised bone prohibits press fit technique, although we have not found this to be necessary. The carpus is reduced and the capsule repaired anatomically with a single running suture. The extensor retinaculum is repaired and the EPL transposed at the surgeon's discretion. The wound is closed in layers with or without suction drainage, and a volar plaster wrist splint applied in neutral position.

The postoperative protocol for midcarpal hemiarthroplasty is relatively straightforward. Active digital, shoulder and elbow range of motion is initiated on postoperative day 1 . The patients return to the office 10 to 14 days after surgery for suture removal. Immobilization is discontinued at that time and patients are enrolled in a supervised program of progressive wrist range of motion (flexion, extension, radial and ulnar deviation, coupled motion including dart-throwing, pronation, supination, and circumduction). Strengthening begins 4 to 6 weeks from the time of surgery and full activity is permitted at 8 weeks. There are no permanent activity restrictions once strength is returned.

\section{Potential Complications}

Wrist hemiarthroplasty assumes the following general risks of implant arthroplasty: wound healing problems, infection, hematoma, aseptic loosening, joint stiffness, nerve or tendon injury, and intraoperative fracture. Potential complications specific to the wrist include extensor adhesions, wrist instability, carpal impingement, and development of symptomatic midcarpal arthrosis. The extent to which these potential complications will play a role in midcarpal hemiarthroplasty is yet to be determined. 


\section{Outcomes}

Restoration of wrist function, while an obvious goal of wrist reconstructive surgery, has been suboptimal in the treatment of painful arthritis of the wrist. Hemiarthroplasty is a novel approach and provides patients with a motion-preserving alternative to procedures that focus on pain relief at the expense of wrist biomechanics and natural motion. This technique restores anatomy by resurfacing the proximal row to preserve the midcarpal joint and the anatomic center of wrist rotation. Carpal kinematics research has demonstrated that this crucial articulation is key to the highly functional dart-thrower's motion of radial-extension to ulnarflexion. ${ }^{43-46,55}$

Wrist hemiarthroplasty is a new technique and current literature is accordingly limited. The only published account of the procedure details the experience of a single surgeon that implanted the proximal component of a Universal 2 Total Wrist (Integra Life Sciences, Plainsboro, NJ) in two young, active patients who were not candidates for total wrist arthroplasty. At 1 and 2 years after surgery, each patient remained pain free, resumed unlimited activity, and maintained range of motion comparable to preoperative levels (flexion arcs of 80 and 69 degrees, respectively). However, there was evidence of hamate erosion in the second case, presumably due to prosthetic impingement, which may compromise long-term success. ${ }^{52}$ Using a cadaveric model, Adams et al showed retention of static capitate alignment after PRC and hemiarthroplasty. Furthermore, their series of 12 patients had no evidence of dislocation or prosthetic complications at early follow-up ("Distal radius arthroplasty with PRC using the Universal 2 implant." International Wrist Investigator's Workshop. 2010).

The KinematX midcarpal hemiarthroplasty was designed to emulate the anatomic contour of the native midcarpal joint, and the corresponding surgical technique developed to preserve the anatomic capsuloligamentous and neural anatomy. The prosthesis preserves radial length and maintains the anatomic COR to provide coupled wrist motion. Replacement of the diseased proximal row provides a painless, stable midcarpal platform for activities requiring combined force and precision.

Over the past 13 months, we have implanted the KinematX hemiarthroplasty in nine patients. Prospective outcome data are currently being collected with Institutional Review Board approval and includes both validated subjective questionnaires and objective measurements. The average age at the time of midcarpal hemiarthroplasty was 43.6 years (range: 23 to 74 years.) Three patients were male and six were female. The dominant hand was involved in six patients. Seven of the nine patients treated were working at the time of surgery, none of which were manual laborers. The average total surgery time was 49 minutes (range: 45 to 60 minutes).

Before surgery, all patients were suffering from chronic, painful degenerative wrist arthritis that limited use of the hand for daily and recreational activities. Posteroanterior and lateral wrist radiographs confirmed the diagnosis in all patients and advanced imaging was not required. The indications for surgery were as follows: SLAC stage 2 (one), SLAC stage 3 (one), SNAC stage 3 (one), posttraumatic osteoarthritis(three), inflammatory arthritis (two; rheumatoid arthritis and psoriatic arthritis), and Keinböck's stage 4 (one). Two patients had preoperative radiographic evidence of early joint space narrowing of the capitolunate articulation. Prior surgery for wrist pain had been performed in two patients (radial styloidectomy for SLAC I, distal radioulnar joint arthroplasty for posttraumatic osteoarthritis). Preoperative range of motion, grip strength, DASH, and Mayo wrist scores are shown

\section{in - Table 2.}

All nine patients were interviewed and examined at regular intervals during their postoperative course. The average length of follow-up was 30.9 weeks (range: 16 to 56 weeks). The mean Mayo wrist score increased from 31.9 (range: 10 to 60 ) preoperatively to 58.8 (range: 30 to 80 ; $p<0.05$ ) and the mean disabilities of the arm, shoulder, and hand (DASH) score improved significantly from 47.8 (range: 22.7 to 70.5 ) preoperatively to 28.7 (range: 0 to 68.2 ) at latest follow-up $(p<0.05)$. The index patient reported a DASH score of 0 at 1 year postoperatively and has resumed golfing regularly. Five of the seven patients that were working before surgery had returned to their regular occupation.

Although there was a trend toward increased motion after surgery, there was no significantly detectable difference in pre- and postoperative range of motion or grip strength measurements (-Table 2). Mean flexion-extension arc was 79 degrees (range: 30 to 130 degrees) and $52.8 \%$ of the opposite hand. Radioulnar deviation averaged 22.9 degrees

Table 2 Pre- and Postoperative Data ${ }^{a}$

\begin{tabular}{|l|l|l|l|}
\hline & Preoperative & Postoperative & Significance $(p)$ \\
\hline Mayo wrist score & $31.9(10-60)$ & $58.8(30-80)$ & 0.006 \\
\hline DASH score & $47.8(22.7-70.5)$ & $28.7(0-68.2)$ & 0.028 \\
\hline FE arc, degrees & $64.6(40-125)$ & $79.3(30-130)$ & 0.362 \\
\hline RD-UD arc, degrees & $16.9(5-50)$ & $22.9(5-37)$ & 0.262 \\
\hline Grip, kg & $16.1(6-35)$ & $18.9(6-38)$ & 0.496 \\
\hline Grip, \% of opposite side & $56.3(30-77.8)$ & $61.7(31-91)$ & 0.501 \\
\hline
\end{tabular}

Note: Includes all patients who underwent hemiarthroplasty (posttraumatic and inflammatory etiologies). Data reported as mean value (range). ${ }^{\mathrm{a}}$ All patients.

DASH, disabilities of the arm, shoulder, and hand; FE, flexion extension; RD-UD, radial deviation-ulnar deviation; kg, kilograms. 
Table 3 Data for Posttraumatic Patients (Inflammatory Arthritis Excluded)

\begin{tabular}{|l|l|l|l|}
\hline & Preoperative & Postoperative & Significance \\
\hline Mayo wrist score & 35 & 67.5 & 0.006 \\
\hline DASH score & 43.2 & 15.9 & 0.006 \\
\hline FE arc, degrees & 58.7 & 90.8 & 0.039 \\
\hline RD-UD arc, degrees & 13.3 & 24.7 & 0.035 \\
\hline Grip, kg & 17.8 & 22.5 & 0.217 \\
\hline
\end{tabular}

Note: Data reported as mean value.

DASH, disabilities of the arm, shoulder, and hand; FE, flexion extension; RD-UD, radial deviation-ulnar deviation; kg, kilograms.

(range: 5 to 37 degrees) and 33.6\%. Mean grip strength was $18.9 \mathrm{~kg}$ (range: 6 to $38 \mathrm{~kg}$ ), which corresponded to $61.7 \%$ of the nonsurgical side.

To date, there was no detectable change in subsequent postoperative radiographs. Neither progressive capitolunate degeneration nor signs of implant loosening were observed.

The only complication identified in our series was postoperative wrist stiffness requiring manipulation under anesthesia in two patients (patient 4 with SLAC wrist and patient 8 with psoriatic arthritis). Their postoperative flexion-extension arcs were restricted to 0 and 5 degrees, respectively, which improved to 50 and 30 degrees after manipulation. Open contracture release was not required. Of note, each of these patients was severely limited in their preoperative motion (45 and 40 degrees), which possibly contributed to their resultant stiffness.

Of specific interest, the two patients in the series with inflammatory arthropathy performed poorly across all points and time. Their postoperative Mayo wrist ${ }^{30,35}$ and DASH (65.9 and 68.2) scores, and grip strengths ( $8 \mathrm{~kg}$ in both) indicate persistent functional limitation not observed in the posttraumatic patients. This prompted us to closely re-examine our data, with the inflammatory patients eliminated from the analysis, and discovered that postoperative increase in motion reached statistical significance. We also observed that the mean postoperative Mayo wrist and DASH scores improved further (-Table 3). Perhaps this is due to unrecognized and persistent inflammatory activity not addressed by the proximal row replacement. While it is not possible to draw a firm conclusion based on the early results of the two patients, we may learn that this procedure is best suited for the patients with posttraumatic degenerative disease.

The limitations of our study must be acknowledged when reviewing our results. Our data are based on single cohort of patients, which presents potential inherent bias. The brief follow-up period precludes any determination of implant durability.

Hemiarthroplasty of the wrist is an exciting and novel technique which is still in its infancy. The procedure is simple and maintains coupled wrist motion through preservation of the midcarpal articulation. The preliminary data in our series support other reports that this technique, in the early postoperative period, appears safe, and has demonstrated encouraging early results. We acknowledge that longitudinal tracking of these patients is required before conclusions can be drawn as to its durability, reliability, and overall benefit. Further study is currently underway to determine whether midcarpal hemiarthroplasty represents an advancement of motion-preserving technology for the treatment of painful radiocarpal arthritis.

\section{Disclaimer}

One or more of the authors receives royalties and consulting fees from Extremity Medical, LLC (Parsippany, NJ 07054).

\section{References}

1 Weiss KE, Rodner CM. Osteoarthritis of the wrist. J Hand Surg Am 2007;32(5):725-746

2 Luo J, Diao E. Kienböck's disease: an approach to treatment. Hand Clin 2006;22(4):465-473, abstract vi

3 Barnard L, Stubbins SG. Styloidectomy of the radius in the surgical treatment of non-union of the carpal navicular. J Bone Joint Surg Am 1948;30A:98-102

4 Smith L, Friedman B. Treatment of ununited fracture of the carpal navicular by styloidectomy of the radius. J Bone Joint Surg Am 1956;38-A(2):368-376

5 Malerich MM, Clifford J, Eaton B, Eaton R, Littler JW. Distal scaphoid resection arthroplasty for the treatment of degenerative arthritis secondary to scaphoid nonunion. J Hand Surg Am 1999;24(6):1196-1205

6 Soejima O, Iida H, Hanamura T, Naito M. Resection of the distal pole of the scaphoid for scaphoid nonunion with radioscaphoid and intercarpal arthritis. J Hand Surg Am 2003;28(4):591-596

7 Ruch DS, Papadonikolakis A. Resection of the scaphoid distal pole for symptomatic scaphoid nonunion after failed previous surgical treatment. J Hand Surg Am 2006;31(4):588-593

8 Neviaser RJ. Proximal row carpectomy for posttraumatic disorders of the carpus. J Hand Surg Am 1983;8(3):301-305

9 DiDonna ML, Kiefhaber TR, Stern PJ. Proximal row carpectomy: study with a minimum of ten years of follow-up. J Bone Joint Surg Am 2004;86-A(11):2359-2365

10 Siegel DB, Gelberman RH. Radial styloidectomy: an anatomical study with special reference to radiocarpal intracapsular ligamentous morphology. J Hand Surg Am 1991;16(1):40-44

11 Linscheid RL, Dobyns JH, Beaubout JW, Bryan RS. Traumatic instability of the wrist.Diagnosis, classification and pathomechanics. J Bone Joint Surg Am 1972;54A:1612-1632

12 Matsuki H, Horii E, Majima M, Genda E, Koh S, Hirata H. Scaphoid nonunion and distal fragment resection: analysis with threedimensional rigid body spring model. J Orthop Sci 2009;14 (2):144-149 
13 Jebson PJ, Hayes EP, Engber WD. Proximal row carpectomy: a minimum 10-year follow-up study. J Hand Surg Am 2003;28 (4):561-569

14 Clayton ML, Ferlic DC. Arthrodesis of the arthritic wrist. Clin Orthop Relat Res 1984;(187):89-93

15 Kobus RJ, Turner RH. Wrist arthrodesis for treatment of rheumatoid arthritis. J Hand Surg Am 1990;15(4):541-546

16 Meads BM, Scougall PJ, Hargreaves IC. Wrist arthrodesis using a Synthes wrist fusion plate. J Hand Surg [Br] 2003;28(6): 571-574

17 Weiss AP, Hastings H II. Wrist arthrodesis for traumatic conditions: a study of plate and local bone graft application. J Hand Surg Am 1995;20(1):50-56

18 Weiss AC, Wiedeman G Jr, Quenzer D, Hanington KR, Hastings H II, Strickland JW. Upper extremity function after wrist arthrodesis. J Hand Surg Am 1995;20(5):813-817

19 Field J, Herbert TJ, Prosser R. Total wrist fusion. A functional assessment. J Hand Surg [Br] 1996;21(4):429-433

20 Carlson JR, Simmons BP. Total wrist arthroplasty. J Am Acad Orthop Surg 1998;6(5):308-315

21 Cohen MS, Kozin SH. Degenerative arthritis of the wrist: proximal row carpectomy versus scaphoid excision and four-corner arthrodesis. J Hand Surg Am 2001;26(1):94-104

22 Mulford JS, Ceulemans LJ, Nam D, Axelrod TS. Proximal row carpectomy vs four corner fusion for scapholunate (Slac) or scaphoid nonunion advanced collapse (Snac) wrists: a systematic review of outcomes. J Hand Surg Eur Vol 2009;34(2): 256-263

23 Vance MC, Hernandez JD, Didonna ML, Stern PJ. Complications and outcome of four-corner arthrodesis: circular plate fixation versus traditional techniques. J Hand Surg Am 2005;30(6):1122-1127

24 Calfee RP, Leventhal EL, Wilkerson J, Moore DC, Akelman E, Crisco JJ. Simulated radioscapholunate fusion alters carpal kinematics while preserving dart-thrower's motion. J Hand Surg Am 2008;33 (4):503-510

25 Arimitsu S, Murase T, Hashimoto J, Yoshikawa H, Sugamoto K, Moritomo $\mathrm{H}$. Three-dimensional kinematics of the rheumatoid wrist after partial arthrodesis. J Bone Joint Surg Am 2009;91 (9):2180-2187

26 Garcia-Elias M, Lluch A, Ferreres A, Papini-Zorli I, Rahimtoola ZO. Treatment of radiocarpal degenerative osteoarthritis by radioscapholunate arthrodesis and distal scaphoidectomy. J Hand Surg Am 2005;30(1):8-15

27 Pervaiz K, Bowers WH, Isaacs JE, Owen JR, Wayne JS. Range of motion effects of distal pole scaphoid excision and triquetral excision after radioscapholunate fusion: a cadaver study. J Hand Surg Am 2009;34(5):832-837

28 Berkhout MJ, Shaw MN, Berglund LJ, An KN, Berger RA, Ritt MJ. The effect of radioscapholunate fusion on wrist movement and the subsequent effects of distal scaphoidectomy and triquetrectomy. J Hand Surg Eur Vol 2010;35(9):740-745

29 Larsen CF, Jacoby RA, McCabe SJ. Nonunion rates of limited carpal arthrodesis: a meta-analysis of the literature.

30 Nagy L, Büchler U. Long-term results of radioscapholunate fusion following fractures of the distal radius. J Hand Surg [Br] 1997;22 (6):705-710

31 Murray PM. Radioscapholunate arthrodesis. Hand Clin 2005;21 (4):561-566

32 Swanson AB. Flexible implant arthroplasty for arthritic disabilities of the radiocarpal joint. A silicone rubber intramedullary stemmed flexible hinge implant for the wrist joint. OrthopClin North Am 1973;4(2):383-394

33 Stanley JK, Tolat AR. Long-term results of Swanson silastic arthroplasty in the rheumatoid wrist. J Hand Surg [Br] 1993;18(3): 381-388
34 Divelbiss BJ, Sollerman C, Adams BD. Early results of the Universal total wrist arthroplasty in rheumatoid arthritis. J Hand Surg Am 2002;27(2):195-204

35 Fourastier J, Le Breton L, Alnot Y, Langlais F, Condamine JL, Pidhorz L. [Guépar's total radio-carpal prosthesis in the surgery of the rheumatoid wrist. Apropos of 72 cases reviewed]. Rev Chir Orthop Repar Appar Mot 1996;82(2):108-115

36 Cobb TK, Beckenbaugh RD. Biaxial total-wrist arthroplasty. J Hand Surg Am 1996;21(6):1011-1021

37 Ward CM, Kuhl T, Adams BD. Five to ten-year outcomes of the Universal total wrist arthroplasty in patients with rheumatoid arthritis. J Bone Joint Surg Am 2011;93(10):914-919

38 Meuli HC. Total wrist arthroplasty. Experience with a noncemented wrist prosthesis. Clin Orthop Relat Res 1997;(342):77-83

39 Dennis DA, Ferlic DC, Clayton ML. Volz total wrist arthroplasty in rheumatoid arthritis: a long-term review. J Hand Surg Am 1986;11 (4):483-490

40 Menon J. Total wrist replacement using the modified Volz prosthesis. J Bone Joint Surg Am 1987;69(7):998-1006

41 Figgie MP, Ranawat CS, Inglis AE, Sobel M, Figgie HE III. Trispherical total wrist arthroplasty in rheumatoid arthritis. J Hand Surg Am 1990;15(2):217-223

42 Menon J. Universal Total Wrist Implant: experience with a carpal component fixed with three screws. J Arthroplasty 1998; 13(5):515-523

43 Crisco JJ, Coburn JC, Moore DC, Akelman E, Weiss AP, Wolfe SW. In vivo radiocarpal kinematics and the dart thrower's motion. J Bone Joint Surg Am 2005;87(12):2729-2740

44 Crisco JJ, Heard WM, Rich RR, Paller DJ, Wolfe SW. The mechanical axes of the wrist are oriented obliquely to the anatomical axes. J Bone Joint Surg Am 2011;93(2):169-177

45 Leventhal EL, Moore DC, Akelman E, Wolfe SW, Crisco JJ. Carpal and forearm kinematics during a simulated hammering task. J Hand Surg Am 2010;35(7):1097-1104

46 Moritomo H, Murase T, Goto A, Oka K, Sugamoto K, Yoshikawa H. In vivo three-dimensional kinematics of the midcarpal joint of the wrist. J Bone Joint Surg Am 2006;88(3):611-621

47 Wilke HJ, Schmidt R, Richter M, Schmoelz W, Reichel H, Cakir B. The role of prosthesis design on segmental biomechanics : Semiconstrained versus unconstrained prostheses and anterior versus posterior centre of rotation. Eur Spine J 2010;(Sep):10

48 Banks SA, Hodge WA. 2003 Hap Paul Award Paper of the International Society for Technology in Arthroplasty.Design and activity dependence of kinematics in fixed and mobile-bearing knee arthroplasties. J Arthroplasty 2004;19(7):809-816

49 Banks SA, Hodge WA. Implant design affects knee arthroplasty kinematics during stair-stepping. Clin Orthop Relat Res 2004; (426):187-193

50 Pearl ML. Proximal humeral anatomy in shoulder arthroplasty: Implications for prosthetic design and surgical technique. J Shoulder Elbow Surg 2005;14(1, Suppl S):99S-104S

51 Shepherd DE, Johnstone AJ. Design considerations for a wrist implant. Med Eng Phys 2002;24(10):641-650

52 Boyer JS, Adams B. Distal radius hemiarthroplasty combined with proximal row carpectomy: case report. Iowa Orthop J 2010;30:168-173

53 Hagert E, Ferreres A, Garcia-Elias M. Nerve-sparing dorsal and volar approaches to the radiocarpal joint. J Hand Surg Am 2010;35 (7):1070-1074

54 Hagert E, Garcia-Elias M, Forsgren S, Ljung BO. Immunohistochemical analysis of wrist ligament innervation in relation to their structural composition. J Hand Surg Am 2007;32(1):30-36

55 Werner FW, Green JK, Short WH, Masaoka S. Scaphoid and lunate motion during a wrist dart throw motion. J Hand Surg Am 2004;29 (3):418-422 